\section{QUALIDADE DE VIDA DE PROFESSORAS DO ENSINO BÁSICO DA REDE PÚBLICA}

\author{
Quality of life of basic education teachers of public schools \\ Calidad de vida de profesoras de la enseñanza básica de la red \\ pública
}

\section{RESUMO}

Objetivo: Avaliar os aspectos associados à qualidade de vida (QV) de professoras do ensino básico da rede pública de Viçosa-MG, buscando relações com a classe econômica. Métodos: Trata-se de um estudo descritivo transversal, desenvolvido entre março e outubro de 2013, realizado com 156 professoras da cidade de Viçosa-MG. Foram aplicados os questionários World Health Organization Quality of Life/BREF e o Critério de Classificação Econômica. Utilizou-se também um questionário contendo tempo de atuação e carga horária semanal de trabalho. Para comparação dos domínios da QV entre as classes econômicas, utilizou-se o teste ANOVA one way, com post hoc de Tukey. Para todos os tratamentos, adotou-se um nível de significância de $p<0,05$. Resultados: As avaliadas apresentaram idade média de $43,88( \pm 10,61)$ anos, carga horária de trabalho semanal de 29,00 $( \pm 10,35)$ horas e tempo de atuação profissional de $16,17( \pm 8,91)$ anos. A maioria das avaliadas se encontrava na classe econômica B1/B2, com 60,3\%. O escore médio da QV geral foi de 68,43 $( \pm 11,69)$ pontos, com maiores valores para os domínios "relações sociais" e "físico". Foi encontrada uma redução na média do domínio "meio ambiente" em relação à menor classe econômica $(\mathrm{p}=0,011)$. Houve correlação fraca e inversa entre o domínio "relações sociais" e a carga horária semanal de trabalho $(\mathrm{r}=-0,16 ; \mathrm{p}=0,031)$. Conclusão: A QV da população estudada foi considerada entre regular e boa, levando-se em conta a escala do protocolo. Houve associação somente entre o domínio "meio ambiente" e a classe econômica.

Descritores: Promoção da Saúde; Qualidade de Vida; Educação; Docentes.

\section{ABSTRACT}

Objective: To evaluate the aspects associated with the quality of life ( $Q \circ L)$ of basic education teachers of public schools of Viçosa, MG, checking for relationships to the economic class. Methods: This is a descriptive cross-sectional study conducted between March and October 2013 with 156 teachers of the city of Viçosa, MG. We applied the World Health Organization Quality of Life/BREF and the Criterion of Economic Classification questionnaires. We also used a questionnaire on the length of employment and the weekly workload. To compare the QoL domains between economic classes, we used the one-way ANOVA with post-hoc Tukey. For all treatments, significance level was set at $p<0.05$. Results: The participants had a mean age of $43.88( \pm 10.61)$ years, weekly workload of $29.00( \pm 10.35)$ hours and length of employment of $16.17( \pm 8.91)$ years. The majority of participants belonged to economic class B1/B2, accounting for $60.3 \%$. The mean score of overall QoL was 68.43 ( \pm 11.69$)$ points, with higher values for the "social relationships" and "physical" domains. There was a reduction in the mean for the "environment" domain in relation to the lower economic class $(p=0.011)$ was found. There was a weak and inverse correlation between "social relationships" domain and the weekly workload ( $r=-0.16 ; p=0.031)$. Conclusion: The QoL of the study population was considered between regular and good, taking into account the protocol scale. There was an association only between the "environment" domain and the economic class.

Descriptors: Health Promotion; Quality of Life; Education; Faculty.
Artigo Original
1) Universidade Federal de Viçosa - UFV Viçosa (MG) - Brasil
Recebido em: 26/02/2015 Revisado em: $13 / 05 / 2015$ Aceito em: 30/05/2015 


\section{RESUMEN}

Objetivo: Evaluar los aspectos asociados a la calidad de vida (CV) de profesoras de la enseñanza básica de la red pública de Viçosa-MG y su relación con la clase económica. Métodos: Se trata de un estudio descriptivo transversal desarrollado entre marzo y octubre de 2013 con 156 profesoras de la ciudad de Viçosa-MG. Fueron aplicados los cuestionarios World Health Organization Quality of Life/BREF y el Criterio de Clasificación Económica. Se utilizo también un cuestionario sobre el tiempo de actuación y la carga horaria semanal de trabajo. Se utilizó la ANOVA one way con el post hoc de Tukey para la comparación de los dominios de la CV entre las clases económicas. Se adoptó el nivel de significación de $p<0,05$ para todos los análisis. Resultados: Las participantes presentaron edad media de 43,88 $( \pm 10,61)$ años, carga horaria de trabajo semanal de 29,00 (土 $10,35)$ horas y el tiempo de actuación profesional de 16,17 $( \pm 8,91)$ años. La mayoría de las participantes era de la clase económica B1/B2 con el 60,3\%. La puntuación media de la CV general fue de 68,43 $( \pm 11,69)$ puntos con mayores valores para los dominios "relaciones sociales" y "físico". Fue encontrada una reducción de la media del dominio "medio ambiente" respecto la más baja clase económica $(p=0,011)$. Hubo correlación débil y inversa del dominio "relaciones sociales" y la carga horaria semanal de trabajo ( $r=-0,16 ; p=0,031)$. Conclusión: La CV de la población investigada fue clasificada entre regular y buena, considerándose la escala del protocolo. Hubo asociación solamente entre el dominio "medio ambiente" y la clase económica.

Descriptores: Promoción de la Salud; Calidad de Vida; Educación; Docentes.

\section{INTRODUÇÃO}

Ao longo da história, transformações econômicas, sociais, políticas e culturais modelaram o ambiente de trabalho. Essa reorganização laboral tem influenciado diretamente na saúde dos profissionais, não sendo diferente no âmbito escolar ${ }^{(1,2)}$.

A docência é uma profissão exposta a alto risco físico e mental ${ }^{(3,4)}$, principalmente devido às condições precárias do ambiente físico de trabalho, às relações conflitantes com alunos e à baixa remuneração, o que vem a intensificar o ritmo e as exigências laborais, repercutindo na qualidade de vida (QV) e saúde dessa populaçãoo ${ }^{(3,5)}$.

A QV é definida pela Organização Mundial da Saúde $(\mathrm{OMS})^{(6)}$ como sendo a autopercepção sobre a sua posição na vida, no contexto da cultura e dos sistemas de valores nos quais vive, e em relação a seus objetivos, expectativas, padrões e preocupações. Tem sido apontada como um importante aspecto multidimensional, constituída por elementos positivos e negativos, associada a múltiplas dimensões das relações humanas ${ }^{(7)}$
Embora existam estudos sobre a saúde e a QV dos professores, ainda são poucas as pesquisas que avaliam os aspectos associando QV com a classe econômica e as condições do trabalho, fora dos grandes centros urbanos. Assim, o conhecimento mais detalhado da influência de diferentes aspectos relacionados à $\mathrm{QV}$ dos docentes é relevante, pois as condições de vida em cidades de pequeno porte e do interior do Brasil podem impactar de forma diferente ao de grandes cidades. Uma vez que ainda são desconhecidas as condições de trabalho de professores da educação básica em muitos contextos brasileiros, e escassas as ações de promoção da saúde nessa população, faz-se necessário considerar a multidimensionalidade do avaliado ${ }^{(5,8)}$. Assim, este estudo objetivou avaliar os aspectos associados à qualidade de vida de professoras do ensino básico da rede pública de Viçosa-MG, buscando relações com a classe econômica.

\section{MÉTODOS}

Trata-se de um estudo descritivo, com um delineamento transversal, realizado na cidade de Viçosa-MG. A coleta dos dados ocorreu de março a outubro de 2013, em oito escolas públicas, com a participação de 156 professoras do ensino fundamental e médio, sendo esse número correspondente a $21,4 \%$ da população de professores registrados na Secretaria de Educação do município no momento do estudo. A seleção da amostra se deu por sorteio aleatório, e como critério de inclusão, a professora deveria ter pelo menos três anos de exercício contínuo como docente do município, independentemente da idade.

O tamanho amostral foi calculado segundo a equação: $\mathrm{n}=\mathrm{P} \times \mathrm{Q} /(\mathrm{E} / 1,96)^{2}$, em que $n$ é o tamanho mínimo da amostra necessária; $P$ é a taxa de prevalência na população; $\mathrm{Q}=100$ - P; e $E$ é a margem de erro amostral tolerado ${ }^{(9)}$. Sendo assim, com $\mathrm{P}=10 \%$, um erro padrão de $5 \%$ e um intervalo de confiança de $95 \%$, chegou-se ao valor de 138 , que equivale ao mínimo de professores necessários. Utilizou-se um $p$ de $10 \%$, visto que estudos realizados em Rio Claro$\mathrm{SP}^{(8)}$ e Florianópolis-SC ${ }^{(10)}$ encontraram valores de $4,7 \%$ e $7,5 \%$, respectivamente, de professores que classificaram sua qualidade de vida como "ruim" ou "muito ruim".

Realizou-se o procedimento de coleta de dados através de uma campanha informática junto à direção, coordenação pedagógica e professoras das oito escolas sorteadas, sendo apresentados todos os objetivos e procedimentos da pesquisa. Após contato inicial e autorizada a intervenção, foi realizado o cadastro das voluntárias que concordaram em participar do estudo.

Utilizou-se questionário padrão do Laboratório de Performance Humana-UFV (LAPEH), que contém dados pessoais, tempo de atuação e carga horária semanal, sendo 
armazenados no software de avaliação física Avaesporte ${ }^{\circledR}$. Após assinatura do termo de consentimento, foi aplicado o questionário Critério de Classificação Econômica (CCEB) $2013^{(11)}$, que classifica a população em função das características domiciliares nas seguintes classes: A1, A2, B1, B2, C1, C2, D e E. Posteriormente, foi entregue o questionário World Health Organization Quality of Life/bref (WHOQOL/breve) ${ }^{(12)}$ que é um instrumento autoaplicável e de autopercepçã̃o. No ato da entrega, foram esclarecidas possíveis dúvidas quanto ao preenchimento dos questionários.

O questionário WHOQOL/breve possui 26 questões, sendo individualmente apresentadas em uma escala crescente de escores que variam de um a cinco, representando as condições da QV. As questões envolvem aspectos diversos da vida cotidiana e abordam quatro domínios da QV: físico, psicológico, meio ambiente e relações sociais. Os resultados são expressos entre 0 e 100 - quanto mais próximo de 100, melhor a qualidade. Dessa forma, um sujeito que apresente valor igual a 50 para determinado domínio pode ser considerado mediano. Todos os cálculos foram realizados de acordo com o estabelecido pelo questionário ${ }^{(12)}$.

Realizou-se o teste de Komolgorov-Smirnov para verificar a normalidade dos dados. Os dados numéricos são apresentados como média, e o desvio-padrão e os categóricos, como percentual. Utilizou-se o teste ANOVA one way, com post hoc de Tukey, para comparação dos domínios entre as classes econômicas. A relação entre idade, tempo de atuação, carga horária semanal e domínios da QV foi feita através do coeficiente de correlação linear de Pearson. Para todos os tratamentos, adotou-se um nível de significância de 5\%. Os dados foram analisados pelo programa estatístico SPSS, versão 17.

Todos os aspectos éticos desta pesquisa respeitaram as determinações do Conselho Nacional de Saúde (Resoluções 466/12), obtendo aprovação pelo Comitê de Ética em Pesquisa da Universidade Federal de Viçosa nº. 070/2012/ CEPH.

\section{RESULTADOS}

A idade média das 156 professoras foi de 43,88 $( \pm 10,61)$ anos, com $56,4 \%$ apresentando 45 anos ou mais. A carga horária de trabalho semanal foi em média de $29,00( \pm 10,35)$ horas, sendo que $48,1 \%$ trabalhavam mais de 24 horas semanais. As professoras tinham tempo de atuação profissional de $16,17( \pm 8,91)$ anos, com $44,2 \%$ das professoras lecionando há 20 anos ou mais. A maioria das avaliadas se encontrava na classe econômica B1/B2, com $60,3 \%$, seguindo pelas classes A1/A2 (17,9\%) e C1/ C2 (14,7\%).

$\mathrm{O}$ escore médio do questionário de avaliação da QV foi de $68,43( \pm 11,69)$ pontos, com maiores valores para os domínios "relações sociais" e "físico". Os mais prejudicados foram "meio ambiente" e "psicológico", com os menores escores (Tabela I).

$\mathrm{Na}$ Figura 1, está apresentada a distribuição percentual das respostas em relação às duas questões gerais, em suas cinco categorias de classificação propostas pelo WHOQOL/ breve. Foram encontrados $7,7 \%$ de respostas "muito ruim e ruim" em relação à autopercepção da QV, e 10,9\% de "muito insatisfeito e insatisfeito" em relação à satisfação com a saúde.

Considerando as demais questões do questionário WHOQOL/breve, os resultados que mais afetaram negativamente, com um percentual considerável de "ruim e muito ruim" ou "insatisfeito e muito insatisfeito", foram oportunidade de atividades de lazer, acesso aos serviços de saúde, satisfação com o sono e recursos financeiros para satisfação das necessidades. Por outro lado, os resultados que afetaram positivamente, com uma elevada prevalência de "bom e muito bom" ou "satisfeito e muito satisfeito", foram os relacionados à capacidade de locomoção, sentido para a vida, satisfação com as relações pessoais e condições de moradia (Figura 2).

A Tabela II apresenta os domínios da QV em relação à classe econômica. Foi encontrada uma redução na média do domínio "meio ambiente" em relação à menor classe econômica $(\mathrm{p}=0,011)$.

Quando realizada a correlação entre os domínios da QV com a idade, o tempo de atuação e a carga horária semanal de trabalho, houve correlação apenas entre o domínio das relações sociais e a carga horária semanal ( $\mathrm{r}=-$ $0,16 ; \mathrm{p}=0,031)$, porém, esta é considerada fraca.

Tabela I - Média e desvio padrão dos escores dos domínios de qualidade de vida (WHOQOL/breve) de professoras do ensino básico da rede pública. Viçosa-MG, 2013.

\begin{tabular}{ll}
\hline Domínios da qualidade de vida & Média $( \pm$ DP $)$ \\
\hline Físico & $70,95( \pm 14,49)$ \\
Psicológico & $68,27( \pm 13,10)$ \\
Relações sociais & $73,18( \pm 15,15)$ \\
Meio ambiente & $61,36( \pm 12,55)$ \\
\hline
\end{tabular}

DP: desvio padrão 


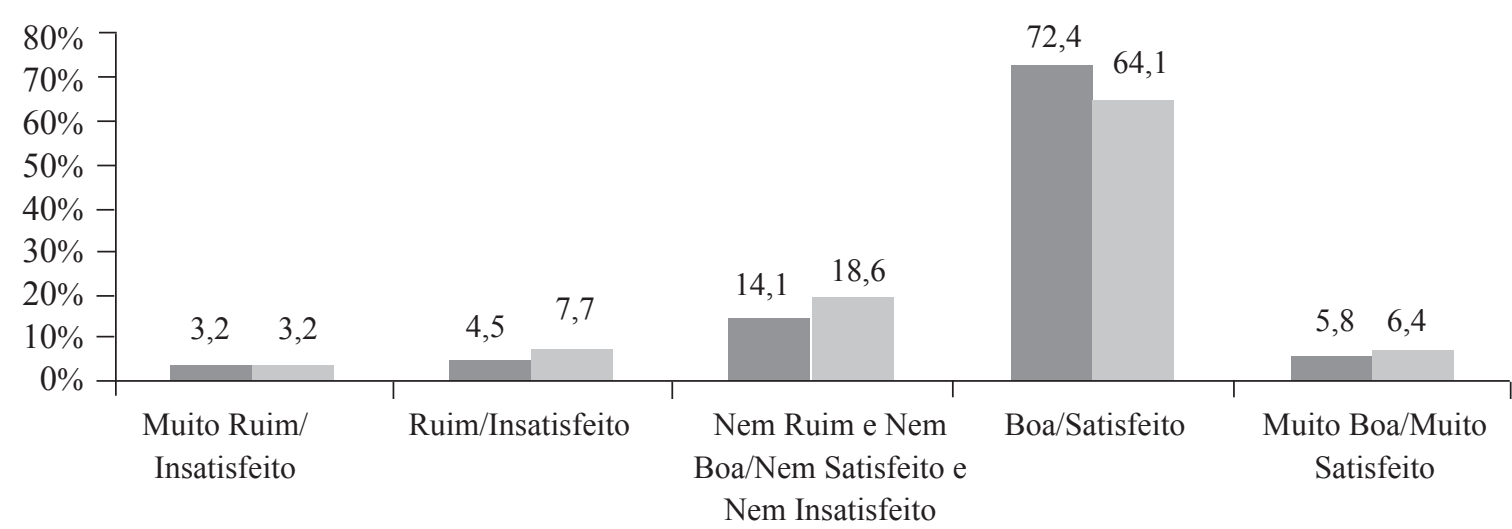

Qualidade de Vida

Saúde

Figura 1 - Autoavaliação da qualidade de vida e saúde de professoras do ensino básico da rede pública. Viçosa-MG, 2013.

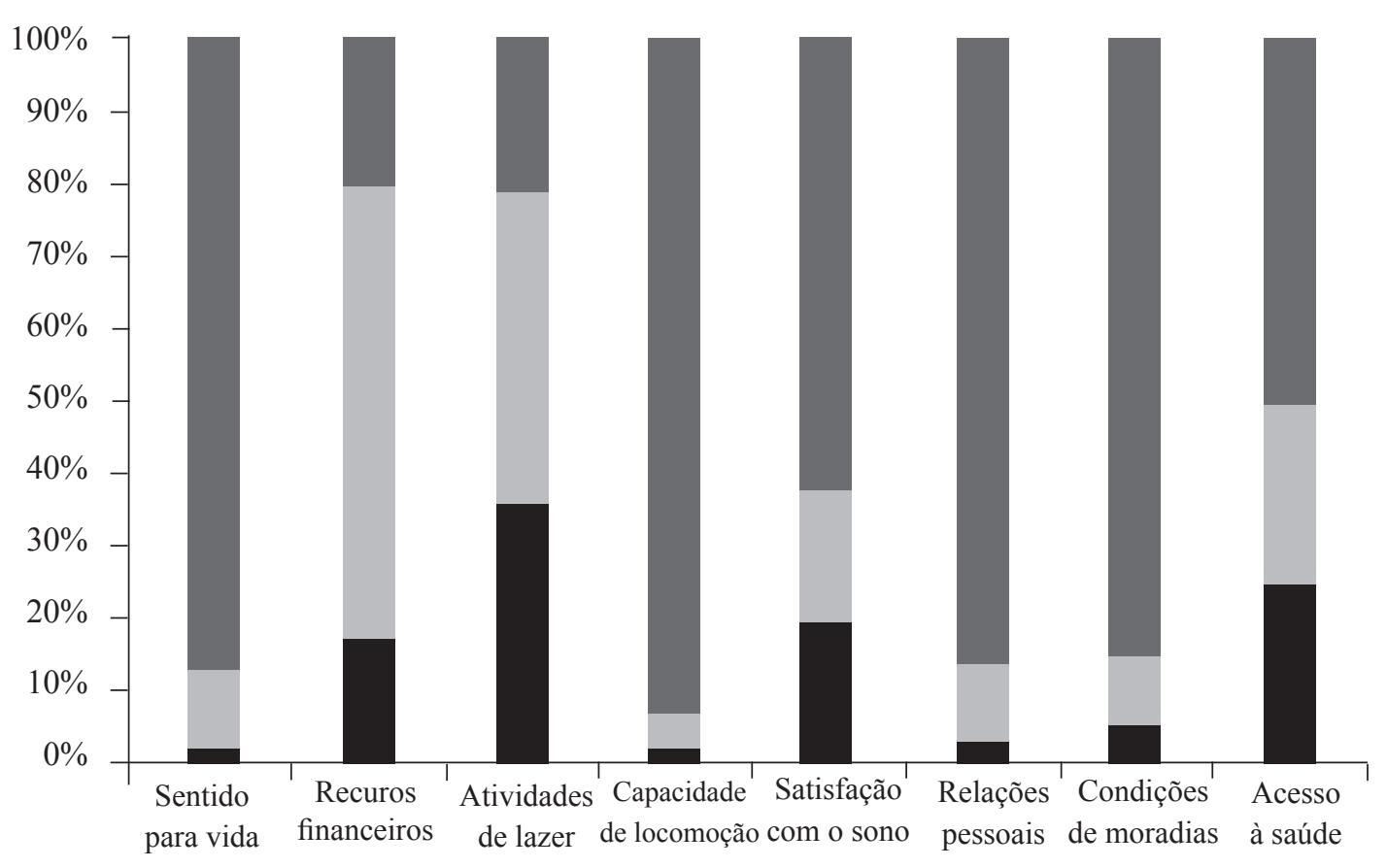

Ruim e Muito Ruim Nem Ruim e Nem Boa Boa e Muito Boa

Figura 2 - Frequência das questões do questionário de qualidade de vida (WHOQOL/breve) de professoras do ensino básico da rede pública. Viçosa-MG, 2013. 
Tabela II - Média e desvio padrão dos escores dos domínios da qualidade de vida (WHOQOL/breve) de professoras do ensino básico da rede pública em função da classe econômica (CCEB). Viçosa-MG, 2013.

\begin{tabular}{|c|c|c|c|c|}
\hline \multirow{2}{*}{ Classe econômica } & \multicolumn{4}{|c|}{ Domínios da qualidade de vida } \\
\hline & Físico & Psicológico & Relações sociais & Meio ambiente \\
\hline $\begin{array}{l}\text { A1 e A2 } \\
(n=28)\end{array}$ & $74,99 \pm 14,25$ & $70,24 \pm 15,27$ & $76,79 \pm 14,05$ & $65,29 \pm 13,35$ \\
\hline $\begin{array}{l}\mathrm{B} 1 \text { e B2 } \\
(\mathrm{n}=105)\end{array}$ & $70,24 \pm 14,21$ & $67,94 \pm 12,79$ & $72,62 \pm 14,69$ & $61,73 \pm 11,45$ \\
\hline $\begin{array}{l}\mathrm{C} 1 \text { e } \mathrm{C} 2 \\
(\mathrm{n}=23)\end{array}$ & $69,25 \pm 15,78$ & $67,39 \pm 11,83$ & $71,38 \pm 18,26$ & $54,89 \pm 14,44^{*} \neq$ \\
\hline$p$ valor & 0,253 & 0,672 & 0,360 & 0,011 \\
\hline
\end{tabular}

* $\mathrm{p}<0,05$ comparado à classe econômica $\mathrm{A} 1$ e A2 (post hoc de Tukey); $\neq \mathrm{p}<0,05$ comparado à classe econômica B1 e B2 (post hoc de Tukey).

\section{DISCUSSÃO}

A amostra apresentou média de $68,43( \pm 11,69)$ pontos na avaliação global da $\mathrm{QV}$, podendo ser considerada entre regular e boa, considerando a escala de zero a cem do WHOQOL/breve. Esse escore foi ligeiramente superior a outros estudos com professores ${ }^{(3,10)}$. Fatores como a carga horária semanal menor, as características de uma cidade do interior em que os deslocamentos casa-trabalho são realizados em trajetos curtos e o período de realização do estudo podem ter influenciado em uma média maior da QV dessa amostra.

A carga horária das professoras avaliadas pode ser considerada como adequada, permitindo, em "teoria", um tempo em atividades que lhes dão prazer, aprimorando sua QV. Isso, por sua vez, tende a provocar um menor nível de estresse no docente, tendo em vista inclusive que já existe uma estabilidade laboral.

Os domínios da QV do WHOQOL/breve ajudam a estabelecer o entendimento de quais aspectos da vida do sujeito influenciam positiva ou negativamente sua autopercepção da QV. No presente estudo, os professores apresentaram maior pontuação no domínio "relações sociais", com 73,18 ( $\pm 15,15$ pontos), e menor pontuação no domínio "meio ambiente", com 61,36 $( \pm 12,55)$ pontos. Pontuações semelhantes foram encontradas em estudo com docentes de Florianópolis-SC. As esferas com menor e maior escore do referido estudo foram, respectivamente, o meio ambiente, com 53,93 pontos, e relações sociais, com 73,10 pontos $^{(10)}$.

Estudos apontam a importância de se possuir uma rede de relações sociais como um fator de proteção contra os efeitos da carga mental e do estresse $\mathrm{e}^{(3,13,14)}$. Os resultados do presente estudo indicam que a amostra é formada por professoras que possuem apoio social, representando bem a influência e/ou efeito do trabalho sobre sua QV.
Os domínios "físico" e "psicológico" afetaram negativamente a avaliação da QV das professoras. Resultados semelhantes foram encontrados em docentes de instituições de ensino superior do Rio Grande do $\mathrm{Sul}^{(13)}$ Essa similaridade se deve, possivelmente, ao fato de as amostras apresentarem características parecidas quanto à idade média, carga horária semanal e tempo de atuação. O domínio "psicológico" é um aspecto que merece atenção, visto que alguns estudos já encontraram um elevado nível de problemas na saúde mental entre esses profissionais ${ }^{(15,16)}$.

A maioria das professoras entrevistadas se mostrou satisfeita com sua condição de saúde (Figura 1), resultado condizente com um estudo envolvendo professores do ensino médio de escolas públicas estaduais, em que $60,2 \%$ disseram estar satisfeitos com a saúde ${ }^{(7)}$.

Esses aspectos devem ser focos de atenção, pois são fatores envolvidos em questões sociais. Deve-se atentar para a melhoria salarial para esse grupo de trabalhares, com vistas a uma maior $\mathrm{QV}$ e, consequentemente, uma melhor atuação profissional ${ }^{(17)}$. Além disso, é interessante a criação de políticas públicas visando um maior acesso aos serviços de saúde e oportunidades de lazer, contribuindo para a promoção da saúde da sociedade em geral.

Quando comparados os domínios da QV em relação à classificação econômica, foi encontrada uma redução na média do domínio "meio ambiente" em relação à menor classe econômica. Na literatura, também foi encontrada relação estatisticamente significante entre os domínios "meio ambiente" e "renda", indicando que a menor renda implicou em baixa pontuação nesse domínio ${ }^{(18)}$. Porém, diferentemente do presente estudo, existem achados que revelam relação também entre o domínio social e a remuneração $0^{(3)}$. Aqueles com menos recursos tendem a possuir uma QV inferior, porém, os dados do presente estudo não comprovam tais afirmações. 
Os domínios da QV podem se relacionar com características específicas do trabalho docente ${ }^{(3,7)}$. Contudo, no presente estudo, somente foi encontrada relação fraca entre o domínio "relações sociais" e a carga horária semanal fraca. Um estudo similar com 203 docentes de instituições de ensino superior do Rio Grande do $\mathrm{Sul}^{(13)}$ encontrou correlação fraca e positiva entre o tempo de serviço e o índice de QV para o domínio "psicológico", e correlação fraca e negativa entre a carga horária e o domínio "social".

Apesar de encontrada fraca correlação entre o domínio "relações sociais" e a carga horária semanal, é importante destacar que elevadas horas de trabalho e tempo de atuação propiciam o aumento de casos de Síndromes de Burnout e prejuízos na saúde mental e física, devido à sobrecarga laboral $^{(3,15,16)}$, restringindo o estabelecimento mais efetivo das relações sociais, com consequente diminuição da QV .

Uma menor carga horária de trabalho implica em um maior tempo disponível para o autocuidado e acesso a bens culturais. Porém, ainda há uma tendência de ocupação desse tempo para desempenhar atividades que complementam a renda familiar, o que vem a contribuir para a sobrecarga de trabalho, com implicações negativas na vida profissional ${ }^{(14,18,19)}$.

Cabe ressaltar que, na atualidade, é presente a multiplicidade de obrigações da mulher no ambiente domiciliar, seja no cuidado com os filhos ou com os afazeres cotidianos da casa ${ }^{(2,5)}$. Além disso, é crescente novas incumbências ao professor, as quais extrapolam o trabalho inerente às atividades tradicionais de ensino ${ }^{(20)}, \mathrm{o}$ que pode vir a ser um fator de agravo à QV das docentes.

A falta de correlação entre idade, tempo de atuação e os domínios da QV pode ser explicada por estar diretamente associada às responsabilidades individuais pela própria saúde e QV, não havendo interferência direta do ambiente de trabalho.

Como limitação do estudo, destaca-se a pequena prevalência de homens na população, o que inviabilizou incluir análise de gênero, sendo interessante a realização de outros estudos entre esse grupo específico. Outra limitação é por se tratar de um estudo transversal, que não proporciona analisar a temporalidade do ano letivo e suas implicações nas condições de saúde e QV dos docentes. Outra abordagem interessante seria a análise de turnos de trabalho, ciclos de ensino diferentes e, por fim, a participação de todos os funcionários do âmbito escolar.

\section{CONCLUSÃO}

Diante dos resultados apresentados, conclui-se que as professoras da educação básica de Viçosa-MG apresentaram escores de "regular" a "bom" na avaliação da
QV. Os aspectos que afetaram negativamente a QV foram os domínios "meio ambiente" e "psicológico". Foi encontrada uma redução na média do domínio "meio ambiente" em relação à menor classe econômica, e uma correlação fraca $\mathrm{e}$ inversa entre o domínio "relações sociais" e a carga horária semanal de trabalho.

Assim, existe a necessidade de planejamento e implementação de ações de promoção da saúde mental, além do desenvolvimento de um ambiente de trabalho adequado, os quais podem influenciar positivamente na $\mathrm{QV}$ dos docentes.

\section{REFERÊNCIAS}

1. Damasio BF, Melo RLP, Silva JP. Sentido de vida, bemestar psicológico e qualidade de vida em professores escolares. Paidéia. 2013;23(54):73-82 .

2. Tabeleão VP, Tomasi E, Neves SF. Qualidade de vida e esgotamento profissional entre docentes da rede pública de Ensino Médio e Fundamental no Sul do Brasil. Cad Saúde Pública. 2011;27(12):2401-8.

3. Pereira ÉF, Teixeira CS, Andrade RD, Bleyer FTS, Lopes AS. Associação entre o perfil de ambiente e condições de trabalho com a percepção de saúde e qualidade de vida em professores de educação básica. Cad Saúde Coletiva. 2014;22(2):113-9.

4. Ceballos AGC, Santos GB. Fatores associados à dor musculoesquelética em professores: Aspectos sociodemográficos, saúde geral e bem-estar no trabalho. Rev Bras Epidemiol. 2015;18(3):702-15.

5. Fernandes MH, Rocha VM, Fagundes AAR. Impacto da sintomatologia osteomuscular na qualidade de vida de professores. Rev Bras Epidemiol. 2011;14(2):27684.

6. World Health Organization (WHO). The World Health Organization Quality of Life Assessment (WHOQOL): position paper from the World Health Organization. Soc Science Med. 1995;41(10):403-9.

7. Penteado RZ, Pereira IMTB. Qualidade de vida e saúde vocal de professores. Rev Saúde Pública. 2007;41(2):236-43.

8. Santos MN, Marques AC. Condições de saúde, estilo de vida e características de trabalho de professores de uma cidade do sul do Brasil. Ciênc Saúde Coletiva. 2013;18(3): 837-46.

9. Lwanga WK, Lemeshow S. Sample size determination in health studies: a practical munual. Geneva, World Health Organization; 1991. 
10. Pereira ÉF, Teixeira CS, Lopes AS. Qualidade de vida de professores de educação básica do município de Florianópolis, SC, Brasil. Ciênc Saúde Coletiva. 2013;18(7):1963-70.

11. Associação Brasileira de Empresas de Pesquisa - ABEP. Dados com base no Levantamento Sócio Econômico 2011-IBOPE. Critério de Classificação Econômica (CCEB). São Paulo: ABEP; 2013.

12. Fleck MPA, Louzada S, Xavier M, Chachamovich E, Vieira G, Santos L, et al. Aplicação da versão em português do instrumento abreviado de avaliação da qualidade de vida "WHOQOL-bref". Rev Saúde Pública. 2000;34(2):178-83.

13. Koetz L, Rempel C, Périco E. Qualidade de vida de professores de Instituições de Ensino Superior Comunitárias do Rio Grande do Sul. Ciênc Saúde Coletiva. 2013;18(4):1019-28.

14. Silveira RE, Reis NA, Santos ÁS, Borges MR. Qualidade de vida de docentes do ensino fundamental de um município brasileiro. Referência 2011;3(4):11523.

15. Pereira ÉFP, Teixeira CS, Andrade RD, Lopes AS. O trabalho docente e a qualidade de vida dos professores na educação básica. Rev Salud Pública. 2014;16(2):22131.
16. Ferreira RC, Silveira AP, Sá MAB, Feres SBL, Souza, JGS, Martins MEBL. Transtorno mental e estressores no trabalho entre professores universitários da área da saúde. Trab Educ Saúde. 2015;13(1):135-55.

17. Alves T, Pinto JMR. Remuneração e características do trabalho docente no Brasil: um aporte. Cad Pesqui. 2011; 41(143):606-39.

18. Azevedo GPGC, Friche AAL, Lemos SMA. Autopercepção de saúde e qualidade de vida de usuários de um Ambulatório de Fonoaudiologia. Rev Soc Bras Fonoaudiol. 2012;17(2):119-27.

19. Costa GLM, Oliveira DA. Trabalho docente no ensino médio no Brasil. Perspectiva. 2011;29(2):727-50.

20. Nardo EL,Schneider MP. Condições de trabalho docente: novas tessituras das políticas de avaliação para a qualidade. Educação. 2014; 39(1):215-28.

\section{Endereço para correspondência:}

Debora Dornelas Ferreira Tavares

Avenida Peter Henry Rolfs, s/n

Campus Universitário

CEP: 36570-900 - Viçosa - MG - Brasil

E-mail: debora.tavaresefi@gmail.com 